Supporting information prepared for publication in Environmental Science and Technology

\title{
Microplastic Fallout in Different Indoor Environments
}

Qun Zhang,,${ }^{\dagger \dagger}$ Yaping Zhao, ${ }^{\ddagger}$ Fangni Du, ${ }^{\dagger}$ Huiwen Cai, ${ }^{\dagger}$ Gehui Wang,${ }^{\S}$ Huahong Shi ${ }^{*}, \dagger$

No. of pages: 2

No. of figures: 1 


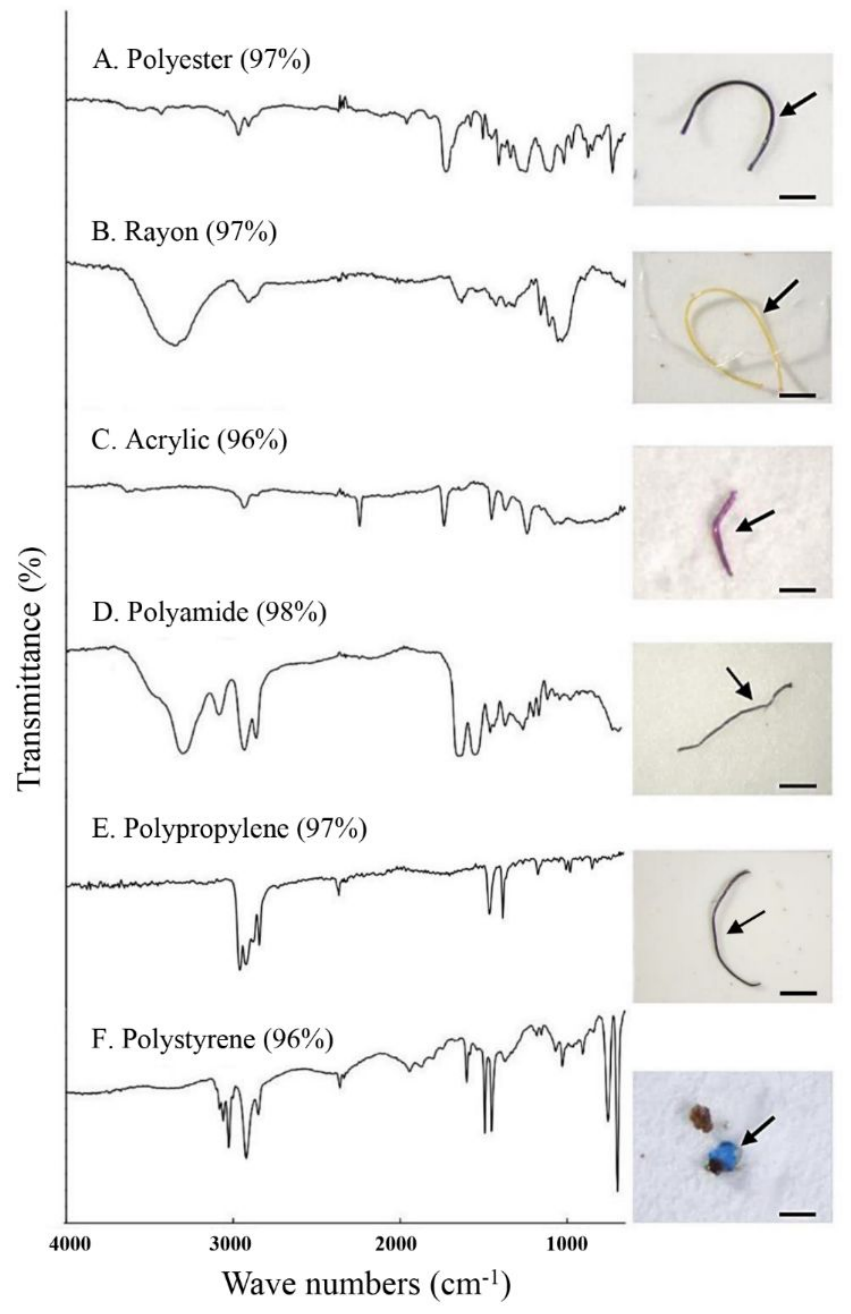

Figure S1. Analysis of microplastics with $\mu$-FTIR. The value in the brackets indicates the matches of the spectra with the standards. The black arrows in the photographs indicate the particles that were identified. Scale bar $=0.1 \mathrm{~mm}$. 